\title{
GEOMETRIC STRUCTURES ON BRANCHED COVERS OVER UNIVERSAL LINKS
}

\author{
KERRY N. JONES
}

\begin{abstract}
A number of recent results are presented which bear on the question of what geometric information can be gleaned from the representation of a three-manifold as a branched cover over a fixed universal link. Results about Seifert-fibered manifolds, graph manifolds and hyperbolic manifolds are discussed.
\end{abstract}

\section{Section 0 - Introduction.}

Closed, orientable three-manifolds admit a variety of universal constructions, that is, constructions by which all manifolds of that class are obtainable, e.g., Heegaard diagrams, surgery diagrams, etc. One of the difficulties faced in three-manifold topology is the decision as to which of the universal constructions is most likely to yield a solution to a particular problem. In this paper, we present several recent results which come from the use of universal links to work on the Thurston Geometrization Conjecture.

More specifically, we present a structure theorem for nonpositively curved Euclidean cone manifolds without vertices, which allows us to deduce which geometries are possible for the pieces of the torus decomposition of certain branched covers, and, in fact, to construct the characteristic submanifold for such covers (in this context, "curvature" refers to a combinatorial condition on the branching indices of a branched cover). We also obtain results concerning hyperbolic structures on negatively curved hyperbolic cone manifolds. Cone manifolds are the natural geometric structure to consider in connection with branched covers, since a cone metric on the base space of a branched covering map may be lifted to the cover.

We also present the negative result that, at least for some universal links, a geometric structure on the cover is not always reflected in the branched covering map itself. More specifically, there exist hyperbolic manifolds, all of whose cone manifold structures arising from branched covering maps over a fixed universal link (the Borromean rings) have some positive curvature.

\section{Section 1 - Universal Links.}

We begin by defining branched covers as well as fixing the notation we will use subsequently

1991 Mathematics Subject Classification. Primary: 57M12, Secondary: 57R15, 57M25.

Key words and phrases. branched cover,universal link,cone manifold. 
Definition. a branched covering map is a continuous map of pairs $\rho:(\hat{M}, \hat{L}) \rightarrow(M, L)$ where $\hat{M}, M$ are $n$-manifolds and $\hat{L}, L$ are $(n-2)$-subcomplexes of $\hat{M}, M$, respectively, such that $\left.\rho^{(}-1\right)(L)=\hat{L}$ and $\rho$ is a covering map when restricted to both $\hat{L}$ and $\hat{M}-\hat{L}$. We say that $\hat{M}$ is a branched cover of $M$, branched over $L$.

For the purposes of this paper, we are interested in the case in which $n=3$ and $\hat{L}, L$ are links. In this case, we may associate to each component $\hat{L}_{0}$ of $\hat{L}$ its branching index, which is the ratio of the degree of $\rho$, restricted to the boundary of a regular neighborhood of $\hat{L}_{0}$ to the degree of $\rho$, restricted to $\hat{L}_{0}$ itself. Equivalently, this is the degree of $\rho$ restricted to a disk in the regular neighborhood of $\hat{L}_{0}$ transverse to $\hat{L}_{0}$. We also note that the branched cover is completely determined by the associated covering map, the restriction of $\rho$ to $\hat{M}-\hat{L}$. We will also use the fact that covering maps of degree $d$ over $M-L$, and thus branched covering maps over $(\mathrm{M}, \mathrm{L})$ are in 1-1 correspondence with conjugacy classes of transitive representations of $\pi_{1}(M-L)$ into $S_{d}$ (that is, representations whose image acts transitively on the set $\{0,1, \ldots, d-1\})$. This monodromy representation associated to a branched covering map is a convenient tool for working with a branched cover. In particular, the branching indices of preimages of a component $L_{0}$ of $L$ are the cycle lengths of the monodromy evaluated on a meridian of $L_{0}$.

Concerning universal links, we make the following formal

Definition. A universal link is a link in $S^{3}$ with the property that all closed, orientable 3-manifolds are representable as branched covers over that link.

It is, of course, by no means obvious that universal links exist at all, but the following theorem tells us that they in fact exist in abundance.

Theorem 1.1 [HLM] and [HLM2]. Any hyperbolic 2-bridge link is universal as are the Borromean rings, the Whitehead link and the knot $9_{46}$.

In fact, at the present time, no hyperbolic links are known to be not universal. The only known obstruction to universality is that a universal link cannot be the connected sum of iterated torus knots or links (see [HLM3]). The problem here is that any branched covering of such a link either contains an essential 2-sphere or is Seifert-fibered.

Y. Uchida has recently shown (see [U]) that hyperbolicity implies universality for another class of links called chains (a chain is a $(\epsilon, \epsilon, \ldots, \epsilon, 2,2, \ldots, 2)$ pretzel link where $\epsilon= \pm 1$ ).

\section{Section 2 - Cone Manifolds and Branched Covers.}

Definition. A Euclidean cone manifold is a metric space obtained as the quotient space of a disjoint union of a collection of geodesic $n$-simplices in $\mathbb{E}^{n}$ by an isometric pairing of codimension-one faces in such a combinatorial fashion that the underlying topological space is a manifold. Hyperbolic and spherical cone manifolds are defined similarly.

One may define cone manifolds in more generality than this (see [Ho], for example), but this definition will suffice for our purposes. Such a space possesses a Riemannian metric of constant sectional curvature on the union of the top-dimensional cells and the codimension-1 cells. On each codimension- 2 cell, the structure is completely described by an angle, which is the sum of the dihedral angles around all of the codimension- 2 simplicial faces which are identified to give the cell. The cone locus of a cone manifold is the closure 
of all the codimension- 2 cells for which this angle is not $2 \pi$ (the Riemannian metric may be extended smoothly over all cells whose angle is $2 \pi$ ). In this paper, we are principally interested in the case where $n=3$ and the cone locus is a link (which must have constant cone angle on each component). We will refer to such cone manifolds as cone manifolds without vertices, meaning no vertices in the cone locus graph with valence other than 2 . The structure at points of lower-dimension cells is not quite so easily described, but we will content ourselves with the observation that some neighborhood of any point in any cone manifold of dimension $n$ is a metric cone on a spherical cone manifold of dimension $n-1$. Thus, each point in a cone manifold determines a spherical cone metric on $S^{n-1}$, after normalization so that the smooth portions of this sphere have curvature +1 . We will refer to this metric on $S^{n-1}$ as the normalized link metric of a point. In the "no vertices" case, this metric is always either the usual smooth metric on $S^{2}$ or a cone metric with two diametrically opposed cone points with equal cone angles.

We should note here that there is a strong connection between cone manifolds and orbifolds (see $[\mathrm{Th}]$ ), namely that orbifolds are cone manifolds with all cone angles of the form $2 \pi / k$ for some integer $k$ ( $k$ is the order of the isotropy of a non-vertex cone point). Orbifolds are (generally) the quotient of a simply-connected manifold by a properly discontinuous, but not necessarily free group action. Note, however, that there need not be such a group action lurking in the background for cone manifolds in general.

One of the principal reasons we are interested in cone manifold structures in connection with universal links is that cone metrics may be lifted to branched covers. More particularly, if $(\hat{M}, \hat{L})$ is a branched cover over $(M, L)$ and $M$ admits a cone metric in which the cone locus is contained in $L$, then we may lift this cone metric to a cone metric on $\hat{M}$ in which the cone locus is contained in $\hat{L}$. The cone angles on the components of $\hat{L}$ are the cone angles on the corresponding components of $L$, multiplied by the corresponding branching indices of the branched covering.

We now have the machinery to make the following

Definition. Let $M$ be a 3-dimensional orbifold with singular set $\Sigma$ a link in $M$ and let $L$ be a link containing $\Sigma$. Then, a branched cover $(\hat{M}, \hat{L})$ over $(M, L)$ is said to be sufficiently branched if all of the branching indices over each component of $L$ are greater than or equal to the order of the isotropy group of that component of L. Similarly, a branched cover is said to be totally insufficiently branched if all of the branching indices over each component of $L$ are less than or equal to the order of the isotropy group of that component.

Note that if we lift the orbifold structure to a sufficiently branched cover, all cone angles are $>2 \pi$ and if we lift to a totally insufficiently branched cover, all cone angles are $<2 \pi$.

Geodesics in a cone manifold are piecewise geodesics (with respect to the underlying constant curvature model) which join at points of the cone locus in such a way as to have an angle of at least $\pi$ between them. Angles between geodesics at a cone point are measured by considering the points of intersection between the two geodesics and the spherical link mentioned earlier. The angle is the distance in the normalized link metric between the two points of intersection. The upshot of this in the "no vertices" case is that if a geodesic encounters a component of the cone locus with cone angle $<2 \pi$, there is no way to continue this geodesic and if a geodesic encounters a component of the cone locus with cone angle $>2 \pi$, there are an infinite number of distinct ways to continue that geodesic. In the "no 
vertices" case, there is also an alternate way of measuring the angle between geodesics, namely by projecting to a totally geodesic disk perpendicular to the component of cone locus at the intersection and measuring the angle in this 2-dimensional cone manifold. As an intuition-building exercise, show that these methods do in fact differ and that in the "no vertices" case, the former method always yields an angle of at most $\pi$, so that geodesic continuation can be recognized by continuation along an angle of exactly $\pi$.

As might be conjectured by considering the Gauss-Bonnet theorem, there is a very strong analogy between cone angle and curvature, with cone angles greater than $2 \pi$ behaving like negative curvature and cone angles less than $2 \pi$ behaving like positive curvature. As an example of this behavior, consider two parallel geodesics in a Euclidean cone manifold that pass on either side of a component of cone locus perpendicular to their common (local) plane. If the cone angle is less than $2 \pi$, the geodesics intersect after passing by the cone geodesic and if the cone angle is greater than $2 \pi$, they diverge after passing by the cone geodesic. This analogy is made precise by the following

Theorem 2.1. Let $M$ be a 3-dimensional cone manifold with no vertices. Then,

(1) if $M$ is spherical and all cone angles are less than $2 \pi, M$ admits a Riemannian metric of positive sectional curvature.

(2) if $M$ is Euclidean and all cone angles are less than $2 \pi, M$ admits a Riemannian metric of nonnegative sectional curvature.

(3) if $M$ is Euclidean and all cone angles are greater than $2 \pi, M$ admits a Riemannian metric of nonpositive sectional curvature.

(4) if $M$ is hyperbolic and all cone angles are greater than $2 \pi, M$ admits a Riemannian metric of negative sectional curvature.

Proof. In each case, one constructs explicitly a smooth metric by altering the cone metric in a tubular neighborhood of the cone locus and verifies the sectional curvature bounds. See [Jo1] for details. Note that this immediately translates into a statement about sufficiently branched and totally insufficiently branched covers over various flavors of orbifolds.

This smoothing technique will be very useful for us in using the machinery of differential geometry to assist in some of our proofs (although direct cone manifold proofs could probably be constructed). Gromov and Thurston in [GT] use this technique in higher dimensions to deduce the existence of negatively curved manifolds which are not hyperbolic. The theorem is probably true even allowing vertices, but it is not clear how to go about explicitly constructing a metric for which the curvature bounds can be verified.

We conclude this section by mentioning the work of Aitchison and Rubinstein (see [AR]) on polyhedral metrics (which are essentially Euclidean cone metrics). The focus of their work is on cone manifolds that do have vertices, but which are built up from pieces of very restricted shapes such as Euclidean cubes and "flying saucers" (generalized cubes which have a cone geodesic with cone angle $2 \pi k / 3$ between diagonally opposed vertices and have $2 k$ square faces). They deduce some very strong results about such cone manifolds when they are nonpositively curved (i.e., when there are no geodesics of length $<2 \pi$ in the normalized metric of any vertex). However, as we will see, it is likely that the manifolds to which their methods and ours both apply are generally Haken manifolds, so there does not appear to be a great deal of useful interplay between the two approaches. 


\section{Section 3 - Surfaces in Branched Covers.}

In this section, we investigate the $\pi_{1}$-injectively immersed surfaces (especially tori) in sufficiently branched covers over Euclidean orbifolds (or, equivalently, Euclidean cone manifolds with all cone angles $>2 \pi$ ).

For the smoothing results of the last section to be truly useful, we must know how that the geometry of the singular metric is in fact closely related to the geometry of the smoothed metric. The next few results tell us that, at least for torus submanifolds, this is indeed the case. Details of these are found in [Jo2]. Most of these results are probably valid for surfaces in general, but the proofs in [Jo2] need tori, and we won't need the additional generality here.

Proposition 3.1. If $M$ is a Euclidean cone manifold with all cone angles $>2 \pi$ and $S$ is a totally geodesic (cone metric) immersed torus, then there is a smooth metric (as in Theorem 2.1) in which $S$ is homotopic to a totally geodesic torus which has the same intersection pattern with the smoothing neighborhood that $S$ does with the cone locus.

Proof. See [Jo2], Lemma 1.3.

Proposition 3.2. If $M$ is a Euclidean cone manifold with all cone angles $>2 \pi$ and $S$ is a $\pi_{1}$-injectively immersed torus in $M$, then $S$ is homotopic to a totally geodesic (cone metric) immersed torus.

Proof. See [Jo2], Lemma 1.4. Here we are using the fact that $\pi_{1}$-injectively immersed surfaces in Riemannian 3-manifolds are homotopic to minimal surfaces [SY] as well as the fact that minimal tori in a 3-manifold of nonpositive sectional curvature must be totally geodesic (a straightforward Gauss equation argument).

Proposition 3.3. If $M$ is a Euclidean cone manifold with all cone angles $>2 \pi$ and $S$ is a totally geodesic surface in $M$, then $S$ is a $\pi_{1}$-injective immersed surface in $M$.

Proof. One simply uses the fact that geodesics in the universal cover of $M$ diverge at least linearly to show that there can be no homotopically trivial closed geodesics in $M$.

We will use these results in the next section, but first we will use Proposition 3.3 to deduce the following rather unfortunate

Theorem 3.4. Let $M$ be a branched cover over $S^{3}$, branched over the Borromean rings with no branching indices equal to one. Then, $M$ is Haken.

Proof. $S^{3}$ admits a Euclidean orbifold structure with singular locus the Borromean rings and all cone angles $\pi$ that arises from "folding" up the faces of a cube as in Figure 3.1. Consider the horizontal plane that cuts through the lines between the faces labelled A and A' (and D and D') one quarter of the distance up the cube. Developing this plane one finds that in all directions the "next" plane is either this same plane or a parallel plane three-quarters of the distance up the cube. These two planes close up to yield an imbedded totally geodesic sphere in $S^{3}$ that only intersects one component of the singular locus (four times). Lifting this sphere to $M$, we find an imbedded totally geodesic surface, which, by Prop. 3.3, must be $\pi_{1}$-injective. It is clearly 2 -sided, and hence incompressible. Since $M$ admits a metric of nonpositive sectional curvature, it must be irreducible by the Cartan-Hadamard Theorem, and thus, $M$ is Haken. 


\section{Figure 3.1}

It may seem a bit curious to have labelled this theorem "unfortunate," since, normally, proving that manifolds are Haken is a cause for rejoicing. However, in this case we have shattered any illusion that geometric structures on manifolds might be always "realizable" by some branched cover representation over any given universal link. More specifically, we have

Corollary 3.5. There exist infinitely many hyperbolic 3-manifolds $M$ for which every representation as a branched cover over the Borromean rings has some branching index equal to 1 (and thus positive curvature in every lifted cone manifold structure).

Proof. Let $M$ be any non-Haken hyperbolic 3-manifold and use Theorem 3.4.

We do, however, also obtain the following two more agreeable corollaries:

Corollary 3.6. Let $M$ be a closed, orientable 3-manifold. Then $M$ is double-branchcovered by a Haken manifold.

Proof. Represent $M$ as a branched cover over the Borromean rings (possible since they are universal) and let $L$ be the index-1 branching locus of $M$ relative to this branched covering. Then, the 2-fold cyclic branched cover of $M$, branched over $L$ is Haken, by Theorem 3.4. This is not a new result, but is a much simpler proof than any of the earlier proofs of this result.

Corollary 3.7. Let $M$ be a branched cover of $S^{3}$, branched over the Borromean rings, with no branching indices equal to 1 or 2. Then, $M$ is hyperbolic.

Proof. By Theorem 2.1 (and the fact that there is a hyperbolic orbifold structure on the Borromean rings with all cone angles $2 \pi / 3), M$ admits a metric of negative sectional curvature. By Theorem 3.4 and Thurston's Hyperbolization Theorem for Haken manifolds, $M$ is hyperbolic. 


\section{Section 4 - Torus Decomposition of Branched Covers.}

In this section, we will consider the torus decomposition of a sufficiently branched cover over a Euclidean orbifold and investigate the possible geometries that may arise. The reader is referred to $[\mathrm{Sc}]$ for a thorough and beautiful discussion of the eight threedimensional homogeneous geometries. It is quite easy to see that if there is a proper decomposition, the Seifert-fibered components must have $\mathbb{H}^{2} \times \mathbb{R}$ or $\mathbb{E}^{3}$ geometry, since a Seifert-fibered manifold with boundary must have one of the three product geometries and the only $S^{2} \times \mathbb{R}$ manifold with boundary is a solid torus, in which case the splitting torus would be compressible. It is also fairly easy to distinguish these two cases geometrically in the following manner: first, homotope the splitting tori to totally geodesic tori (or possibly Klein bottles) using standard minimal surface techniques and the theorems of the previous section. Then, if a Seifert-fibered component of the torus decomposition contains a component of the cone locus, it must have $\mathbb{H}^{2} \times \mathbb{R}$ geometry, else it must have $\mathbb{E}^{3}$ geometry (see [Jo1], essentially this is a corollary of a fact, proven there, that a closed Euclidean 3-manifold cannot admit a Euclidean cone manifold structure with all cone angles greater than $2 \pi$ ). Also in [Jo1], a straightforward growth of groups argument is used to show that a closed Seifert-fibered Euclidean cone manifold with cone angles $>2 \pi$ must have $\mathbb{H}^{2} \times \mathbb{R}$, Nil, or $\widetilde{S L_{2} \mathbb{R}}$ geometry.

Some more recent results, however, give improvements on this by eliminating Nil from the list and by giving an easy way to construct the torus decomposition of a Euclidean cone manifold with all cone angles $>2 \pi$. Details for these results may be found in [Jo3].

First, a structure theorem for such manifolds:

Theorem 4.1. Let $M$ be a closed, orientable 3-dimensional Euclidean cone manifold with no vertices and all cone angles $>2 \pi$. Then there is a canonical compact 2-complex $C$ in $M$ such that

(1) the components of the complement of $C$ (denoted by $M_{1}, \ldots, M_{n}$ ) are each the interior of a compact Seifert-fibered manifold (possibly with boundary)

(2) each $M_{i}$ may be given a convex Euclidean cone metric

(3) $M$ is homotopically atoroidal if and only if each $M_{i}$ is an open solid torus

This canonical 2-complex is easily constructed from the cone manifold by considering product neighborhoods of cone geodesics and looking at the intersections of maximal such neighborhoods. The Seifert-fibration on each of the $M_{i}$ is such that any cone geodesics that intersect the $M_{i}$ are in fact fibers of the Seifert-fibration. This is another example of just how "canonical" the notion of a Seifert-fibration seems to be: there are a number of results now of the general form "If a Seifert-fibered manifold admits structure X, then it admits a structure $\mathrm{X}$ which is nice with respect to the fibration." Some of the known replacements for "structure X" are "incompressible surface," "essential lamination" and "Euclidean cone metric with cone angles $>2 \pi$ " (see [Br] for the essential lamination result).

One of the most useful aspects to this structure theorem, however, is that the boundary tori of the $M_{i}$ which are not solid tori (after pulling them in slightly along a collar neighborhood) form a collection of tori which contains the characteristic tori of JacoShalen/Johannson. Furthermore, the only way in which "extra" tori arise is from the fact that some Seifert-fibered spaces may be fibered in more than one way and thus the fiberings that are constructed in the structure theorem might be incompatible across a given 
torus but there might be another way to fiber some component of the complement of this collection of tori so that there would be compatibility across a torus, and so it could be removed. However, the only spaces that actually arise in the decomposition that may be fibered in multiple ways are $I$-bundles over the torus and Klein bottle (see [Sc] again) so this situation is easily handled to compute the actual torus decomposition. Furthermore, all the Seifert-fibered components of the complement of this collection of tori either have negatively curved base orbifold or are actually Euclidean, so we have the elimination of Nil as claimed above.

Another interesting corollary of this theorem is the following: suppose $M$ is a Euclidean cone manifold with all cone angles $>2 \pi$. Suppose further that $M$ admits a $\pi_{1}$-injective immersed torus but no incompressible tori. We use the structure theorem to assert that $M$ must be Seifert-fibered, since if $C$ is nonempty, any component of its complement that isn't a solid torus gives rise to a nontrivial torus decomposition as above, but all of the components being solid tori forces $M$ to be homotopically atoroidal. Thus, the only possibility is that $C$ is empty, forcing $M$ to be Seifert-fibered. Thus, we recover the recent result of Casson and Gabai (independently, see [Ga]) that irreducible non-Haken 3manifolds containing $Z \oplus Z$ subgroups in their fundamental groups must be Seifert-fibered.

For convenience, we collect these in the following

Corollary 4.2. If $M$ is a Euclidean cone manifold satisfying the hypotheses of Theorem 4.1, then

(1) if $M$ admits a $\pi_{1}$-injective torus but no incompressible torus, $M$ must be Seifertfibered

(2) the collection of boundary-parallel tori in each non-solid torus component of $M_{i}$ forms a collection of tori containing the Jaco-Shalen/ Johannson characteristic tori

(3) if $M$ is Seifert-fibered, it must have $\mathbb{H}^{2} \times \mathbb{R}$ or $\widetilde{S L_{2} \mathbb{R}}$ geometry

\section{Section 5 - Hyperbolic Structures on Branched Covers.}

Thus far, we have discussed primarily Euclidean cone manifolds. We will conclude by turning briefly to hyperbolic cone manifolds. Here, one of the key questions is that of deformability. That is, how far can one deform a hyperbolic cone manifold without running into some degeneracy or other. Hyperbolic cone manifolds can always be deformed locally, but there are several kinds of degeneracy that may eventually arise (see [Ho] for a more detailed discussion). The possible kinds of degeneracy are

(1) volume tending to zero

(2) developing a cusp along a submanifold which is becoming Euclidean

(3) the cone locus "bumping into itself" (that is, changing its combinatorial type

It has long been conjectured that cone angles may always be decreased. One reason for this conjecture (other than the fact that no counterexamples are known) is that when the cone angles are being decreased, the first kind of degeneracy cannot happen - volume always increases when all cone angles are decreasing. Furthermore, as the cone angles are decreased, the existence of the submanifolds that are "becoming Euclidean" can often be ruled out on other grounds. In particular, if we have a hyperbolic cone manifold with all cone angles $>2 \pi$, neither of the first two kinds of degeneracy can take place as we deform 
all cone angles toward $2 \pi$ since the manifold is known to be atoroidal and aspherical (all of the submanifolds along which a cusp would develop must be essential spheres and tori). The third kind of degeneracy has proven to be quite difficult to rule out a priori, and very little progress in this direction has been made (although some recent progress has been announced by Hodgson and Kerckhoff [unpublished]).

However, if one is seeking to show that hyperbolic cone manifolds with cone angles $>2 \pi$ are in fact hyperbolic, there are other means besides simply deforming the cone metric. One may also try using the smoothing results above to obtain a metric of pinched negative sectional curvature and attempt a deformation of this metric toward constant sectional curvature. Of course, this is an extremely difficult problem as well, and is in fact known to be impossible in dimensions higher than 3. However, there is a result of Tian [Ti] on deformation of negatively curved metrics to Einstein metrics (which in dimension 3 have constant sectional curvature). That does enable us to at least deduce a sort of asymptotic result. This theorem (details of which are found in [Jo4]) essentially says that for a fixed hyperbolic orbifold, we can find a constant such that if the maximum and minimum branching indices of a branched cover have a ratio less than this constant, the branched cover must be hyperbolic. This is the essence of the theorem, although there are some unfortunate details that complicate its statement - the "constant" is not quite constant, but depends on the number of components of the branching locus that don't have the minimum branching index.

The actual statement of the theorem is

Theorem 5.1. Let $(M, L)$ be a 3-manifold and a link such that $M-L$ admits a hyperbolic metric of finite volume. Let $L_{1}, \ldots, L_{q}$ be the components of L. Let $(\hat{M}, \hat{L})$ be a branched cover over $(M, L)$ with minimum branching index $n_{i}$ over $L_{i}$ and maximum branching index $N_{i}$ over $L_{i}$. Denote by $Q_{i}$ the number of components of branching locus over $L_{i}$ with branching index not equal to $n_{i}$ (counted with the appropriate multiplicity in the case of longitudinal wrapping). Then, there exist integers $\left(m_{1}, \ldots, m_{q}\right)$ and functions $\left(K_{1}, \ldots, K_{q}\right)$ with $K_{j}: \mathbb{Z} \rightarrow \mathbb{R}$ and $K_{j}(i)>1$, only depending on $(M, L)$, such that $(\hat{M}, \hat{L})$ admits a hyperbolic metric if $n_{i} \geq m_{i}$ and $N_{i} \leq n_{i} K_{i}\left(Q_{i}\right)$ for all $i=1, \ldots, q$.

Proof. One takes a particular smoothing of the lifted cone metric on $\hat{M}$ for which explicit bounds on the sectional curvature may be computed, and then applies Tian's theorem to deduce the existence of a nearby Einstein metric (again, see [Jo4] and [Ti] for details).

Although this result is far from ideal (we have no idea what order of magnitude the $m$ 's and $K$ 's have, so we cannot use the result to show that any given cover is hyperbolic), it is the first result giving combinatorial conditions on the branched covering map under which irregular branched covers of hyperbolic links must be hyperbolic (regular branched covers are dealt with by the Thurston Geometrization Theorem for Orbifolds and the case in which all branching indices are equal is dealt with by the Hyperbolic Dehn Surgery Theorem).

\section{REFERENCES}

[AR] I.R. Aitchison and J.H. Rubinstein, An introduction to polyhedral metrics of non-positive curvature on 3-manifolds, LMS Lecture Note Series 151 (1990), 127-162. 
[Br] M. Brittenham, Essential Laminations in Seifert-Fibered Spaces, thesis, Cornell University, 1990.

[GT] M. Gromov and W. Thurston, Pinching constants for hyperbolic manifolds, Invent. Math. 89 (1987), 1-12.

[Ga] David Gabai, Convergence groups are Fuchsian groups, preprint

[HLM1] H.M. Hilden, M.T. Lozano, and J.M. Montesions, The Whitehead link, the Borromean rings and the Knot $9_{46}$ are universal, Collec. Math. 34 (1983), 19-28.

[HLM2] _ _ On knots that are universal, Topology 24 (1985), 499-504.

[HLM3] _ Non-simple universal knots, Math. Proc. Camb. Phil. Soc. 102 (1987), 87-95.

[Ho] Craig Hodgson, Geometric structures on 3-dimensional orbifolds: notes on Thurston's proof, preprint.

[Jo1] Kerry N. Jones, Cone manifolds in 3-dimensional topology: applications to branched covers, thesis, Rice University (1990).

[Jo2] , Injectively immersed tori in branched covers over the figure eight knot, Proc. Edinburgh Math. Soc. (to appear).

[Jo3] - The structure of closed nonpositively curved Euclidean cone 3-manifolds, preprint, 1992.

[Jo4] _ Hyperbolic structures on branched covers over hyperbolic links, preprint, 1992.

[SY] R. Schoen and Shing-Tung Yau, Existence of incompressible minimal surfaces and the topology of three dimensional manifolds with non-negative scalar curvature, Ann. of Math. 110 (1979), $127-142$.

[Sc] Peter Scott, The geometries of 3-manifolds, Bull. LMS 15 (1983), 401-487.

[Th] William P. Thurston, The geometry and topology of three-manifolds, Princeton University Course Notes, 1979

[Ti] Gang Tian, A pinching theorem on manifolds with negative curvature, preprint.

[U] Y. Uchida, Universal chains, Kobe J. Math. 8 (1991), 55-65.

The University of Texas, Austin, TX 78712 